\title{
Cognitive Emotion Regulation, Anxiety, and Depression in Patients Hospitalized with COVID-19
}

\author{
Daria V. Dovbysh ${ }^{*}$ Maria G. Kiseleva \\ Sechenov University, Moscow, Russia \\ * Corresponding author. E-mail: dashadovbysh@gmail.com
}

Background. A number of studies from different countries have been devoted to studying the psychological state of patients during the COVID-19 pandemic. In addition to the severity of the symptoms of the disease itself, the situation of uncertainty can negatively affect the patients' psychological well-being.

Objective. Our research aimed to explore ways for patients with COVID-19 to regulate their emotional state during hospitalization, and how they can reduce symptoms of depression and anxiety.

Design. The research involved 127 people hospitalized due to confirmed COVID-19: 67 men (52.8\%) and 60 women $(47.2 \%)$, ages 19 to 77 years $(M=43.34$, $\mathrm{Me}=42, \mathrm{SD}=11.81$ ). We used a set of questionnaires which included the Beck Depression Questionnaire; the Generalized Anxiety Disorder scale; the Perceived Social Support Questionnaire (22-item); the Cognitive Emotion Regulation Questionnaire; and the Dembo-Rubinstein self-assessment scales.

Results. Twenty-five and four-tenths percent (25.4\%) of the participants had severe symptoms of anxiety, and $24.13 \%$ had symptoms of depression. Women showed higher symptoms of depression than men. ANOVA showed no significant differences in the use of emotion regulation strategies in patients being hospitalized at different intervals, or in patients of different age groups. Factor analysis made it possible to distinguish three patterns of emotion regulation: 1) adaptive cognitive change; 2 ) fixation on negative experiences; and 3) deflection of responsibility. Significant positive correlations were found between symptoms of depression and anxiety, and coping by fixation on negative experience only.

Conclusion. Although various means of cognitive emotion regulation by patients hospitalized with COVID-19 are currently being presented, these strategies are not associated with significant reductions in their symptoms of depression and anxiety.
Keywords: anxiety; depression; pandemic; COVID-19; regulation of emotion; coping with the disease 


\section{Introduction}

The COVID-19 pandemic, which began in 2020, has clearly become a challenge for not only health professionals but all people, since all are directly or indirectly affected by quarantine measures and the threat of the disease to their daily lives. A large number of psychological studies have been devoted to studying the peculiarities of the response to the pandemic among residents of different countries and regions, including both those where the spread of COVID-19 took place rapidly, and those where the number of cases grew much more slowly. Most research has emphasized the emergence of symptoms of anxiety and depressive disorders at different stages of quarantine measures, as well as high levels of stress (Petzold et al., 2020; Wang et al., 2020). Meta-analysis data has shown an average depression rate of $33.7 \%$ and anxiety rate of $31.9 \%$ (Salari et al., 2020).

Social support, high personal self-efficacy, clear and understandable information on how to get help, lack of physiological symptoms, and lack of continuous monitoring of new cases (among other conditions), as well as adherence to certain preventive measures, have been identified as protective factors significantly associated with reduced anxiety (Domínguez-Salas et al., 2020; Petzold et al., 2020). A number of authors have noted that the severity of symptoms of depression and anxiety has not decreased during the pandemic and remains at a fairly high level (Wang et al., 2020). There is also evidence of a growing rate of suicide among both the total population and patients (Sher, 2020). In addition, studies have confirmed the generally known pattern of greater severity of symptoms of depression and anxiety in women than in men; the COVID-19 situation is not an exception (Petzold et al., 2020).

The study of COVID-19 responses among patients with a confirmed diagnosis is a special task for both the scientific community and practicing psychologists and physicians. However, even the meta-reviews of publications on the COVID-19 issue, which analyzed the response of the general population, vulnerable populations, and health workers, do not always contain information about what happened to the patients (Rajkumar, 2020). These meta-reviews include both overview theoretical articles and case analyses that assert the need for psychological support for patients with COVID-19. They also show that anxiety and depressive symptoms, common among hospitalized patients, in cases of COVID-19 can be enhanced by specific somatic sensations, such as respiratory insufficiency and cardiovascular complications, as well as by distance from social support (Epstein et al., 2020; Xiang et al., 2020).

At the same time, other authors note that, regardless of the success of medical protocols for treating COVID-19, psychological support of patients may reduce the severity of some somatic symptoms associated with the disease (Renjun et al., 2020). Chinese colleagues also talk about a possible link between patients' selfreported depression and an immune response; in this case, psychological support is especially significant, as it is potentially associated with the effectiveness of drug treatment (Yuan et al., 2020). There is also evidence showing the effectiveness of online training aimed at reducing anxiety and depression in patients with COVID-19 through the use of mindfulness techniques, breathing techniques, "refuge" skills (techniques for creating a safe space in the patient's imagination), and the 
"butterfly hug method" (a physical technique aimed at reducing the intensity of emotions) (Wei et al., 2020). But many studies only assert the effectiveness of these techniques, without explaining their mechanisms.

In this regard, we have focused our study on analyzing the means of emotional regulation and coping with the disease being used by hospitalized patients; we also thought it important to investigate the relationship between the strategies used and the severity of symptoms of depression and anxiety. As the main construct, we selected the cognitive regulation of emotions model developed by Garnefski and Kraaij (2007).

Over the last decade, the study of emotion regulation as a separate psychological phenomenon has grown rapidly and become popular. This interest is largely due to the fact that most authors agree that the regulation of emotions is an important link in the preservation and restoration of mental health (Gross, 2013; Sapolsky, 2007). In its most general form, emotion regulation can be defined as "external and internal processes responsible for collecting, evaluating and changing emotional responses, especially their intensity and length over time in order to achieve certain goals" (Thompson, 2008). From the point of view of Garnefski and her colleagues, such a wide definition includes both coping strategies and controlling behavior. Within Garnefski's model, the division of coping strategies into behavioral and cognitive seems more appropriate than classification of "effective" - "not effective" or "emotionally oriented" - "problem-oriented," since thought processes and behavior are different processes occurring at different points in time (Garnefski, Kraaij, \& Spinhoven, 2000).

In researching how hospitalized patients respond to COVID-19, it seemed reasonable to concentrate on the precise cognitive methods they used to regulate their emotional state, since the very fact of hospitalization in connection with an infectious disease places a person in conditions where the implementation of many familiar behavioral coping strategies (for example, seeking help from a specialist; sports and physical activity; travelling; meeting with friends; massage or bath, etc.) is impossible. In this regard, cognitive strategies turn out to be the "first line of defense" that helps a person survive and accept having a serious and unknown disease.

Therefore, the focus of our attention was the study of the main strategies for cognitive regulation of emotions. We identified the following strategies: self-blame; acceptance; rumination; positive refocusing; refocus on planning; positive reappraisal; putting into perspective; catastrophizing; and blaming other patients with COVID-19 and their correlations with the symptoms of depression and anxiety.

A meta-analysis of modern experimental studies of various strategies of emotional regulation has shown that it is impossible to draw an unambiguous conclusion about which strategies for emotional regulation work and which do not (Gross \& Thompson, 2007). Strategies that have been successful in one case may not lead to success in another, so it is always necessary to evaluate them in the context of a specific situation, and judge how much of the emotional response corresponds to what is happening, and whether another more adaptive reaction is possible in the situation. This explains why currently it is extremely important to consider the patients' existing strategies specifically in relation to the COVID-19 pandemic: the fact that a previous pattern of emotion regulation helped to successfully cope with stressful events does not mean that this pattern will be productive in this pandemic. 


\section{Methods}

\section{Participants}

Our study involved 127 respondents (52.7\% men, $47.2 \%$ women) who voluntarily agreed to participate in the research. The age of participants was from 19 to 77 years, (mean age 43.34, median age 42, standard deviation 11.91). All participants were hospitalized due to a confirmed diagnosis of COVID-19 at the University Hospital of Sechenov University and had been in the hospital for 10-14 days at the time of participation in the study. All participants lived in Moscow. Unfortunately, we do not have additional medical data on the severity of symptoms, comorbidities, etc.

\section{Materials}

\section{Questionnaires}

1. The Beck Depression Inventory-II (BDI-II) (Beck, Steer, \& Brown, 1996) in Russian adaptation by Tarabrina (Tarabrina, 2001). BDI-II was designed to assess the presence and severity of depressive symptoms in a patient over the previous 14 days. The questionnaire consists of 21 statements, with each answer being scored on a scale of 0 to 3 ; total scores thus range from 0 to 63 . Values from 0 to 13 mean no depression \minimal depression; 14 to 19, mild depression; 20 to 28, moderate depression; and 29 to 63, severe depression (according to international cut-off scores). The test has high internal consistency $(\alpha=0.86)$ (Tarabrina, 2001). We also separately assessed scores on cognitive-affective and somatic sub-scales as part of this questionnaire (internal consistency $\alpha=0.79$ for both sub-scales).

2. The Generalized Anxiety Disorder scale (GAD-7) (Spitzer, Williams, \& Kroenke et al., 2006). This scale measures the severity of anxiety symptoms in patients over the previous two weeks. Answers to each item are scored from 0 to 3 points, with total scores ranging from 0 to 21 . Scores from 0 to 4 points indicate the minimum level of anxiety; 5 to 9, moderate; 10 to 14, medium; and $\mathrm{m} 15$ to 21 , a high level of anxiety, according to international cut-off scores (Spitzer, Williams, \& Kroenke et al., 2006). The internal consistency of the GAD-7 is also high $(\alpha=0.92)$

3. Perceived Social Support Questionnaire (F-SOZU-22) (G. Sommer \& T. Fydrich, 1993) in Russian adaptation by Kholmogorova, Garanian, and Petrova (2003). This questionnaire measures general perceived social support with a 5-point scale ranging from 1 (not true at all) to 5 (very true). We used scales of social support and satisfaction with received social support. The minimum and maximum scores on the first scale are 12 and 60, respectively (12 questions included); and on the second, 2 and 10 points, respectively ( 2 questions included). The internal consistency of the F-SOZU-22 was $\alpha=0.89$.

4. Cognitive Emotion Regulation Questionnaire (CERQ) (Garnefski \& Kraaij, 2007), in Russian adaptation by Rasskazova, Pluzhnnikov, and Leonova (2011). We asked respondents to answer about how they cope with the difficult situation of hospitalization due to COVID-19. The questionnaire emphasizes the following scales: self-blame, acceptance, rumination, positive refocusing, refocus on planning, positive reappraisal, putting into perspective, catastrophizing, and blaming others. The questionnaire consists of 36 statements (4 items for each scale). The answer to each question is given on a 5 -point scale: $1=$ never and $5=$ almost always. Scores on each of the 9 scales range from 4 to 20. The internal consistency (Cronbach's alpha) for different scales varies from 0.74 to 0.83 . 
5. A modified version of the Dembo-Rubinstein technique, which included such scales as health (DR Health), luck (DR Luck), stress tolerance (DR Stress tolerance), happiness (DR Happiness), and sociability (DR Sociability) (Rubinshtejn, 2010).

In this version of the technique, the subjects were asked to evaluate the severity of the presented qualities on a 10 -point scale, where $1=$ completely absent and $10=$ present as much as possible. The instructions called for the patient to answer one question for each scale. Here are sample scales that assess the parameters of well-being or personality traits that are important for each person: "Imagine that all people on Earth are placed on these scales, and people with the lowest intensity of this quality are located at rating 1, and people with the highest severity are at 10 . Please determine your place on these scales." The scores on each scale ranged from 1 to 10 .

6. The socio-demographic survey, which included questions about age, sex, education, subjective severity of symptoms, and satisfaction with the quality of care, as well as about the needs for other forms of assistance (work with a psychologist, priest, closer contact with a doctor, etc.).

\section{Procedure}

Patients who had been at the clinic between 10 and 14 days were asked to fill out an online form (using Google Forms service); a link to the form was sent to the mobile phone of those who agreed to take part in the research. Participation in the study was voluntary. All participants completed the proposed questionnaires anonymously. Patients were advised that participation in the study would not affect the quality of the care they received., The participants did not receive feedback on the results of the study, but were informed about the possibility of seeking psychological help on the helpline.

We conducted our research between April 25, 2020 and May 31, 2020. At that time, strict self-isolation measures were already in force in the city of Moscow (work and studies via remote format), and patients and members of their families had to stay at home and regularly confirm their location using an online application. For hospitalized patients, such self-isolation meant the impossibility of communicating with relatives and friends face to face and sharing items with each other, as well as having a more limited contact with medical personnel (shorter in time; wearing mask and protective suit), who, according to the instructions, had to wear special protective clothing

We identified three periods of hospitalization: the First Stage was from April 25 to May 1; the Second Stage from May 1 to May 12; and the Third Stage from May 12 to May 31. We were guided by the following reasoning: 1) Because workers in Russia traditionally get several days off between May 1 and 12 due to public holidays, which often involve a large number of mass events (mass holidays, trips with family and friends), this period was important for assessing the severity of depressive and anxiety disorders for those patients whose hospitalization occurred on these weekends; 2) Patients hospitalized during the early stages of the pandemic reported a greater level of uncertainty and less understanding of the health system in the questionnaire. We assumed that an early date of hospitalization would be asso- 
ciated with greater difficulty in orienting in a stressful situation and using other coping strategies than if they were hospitalized at the end of the research period, which was right before quarantine measures were removed. During the first stage, 15 patients were interviewed (11.8\% of participants); during the second, 70 people (55.1\% of participants); and during the third, 42 people (33.1\% of participants).

We divided the study participants into groups by gender: $52.7 \%$ of participants were men and $47.2 \%$ were women.

We also identified three age groups: $19-35$ years old (32 people, $25.2 \%$ of participants); $35-50$ years old (65 patients, 51.2\%); and 50-77 years old (30 people, $23.6 \%$ of patients).

\section{Results}

Table 1 provides descriptive statistics of the main parameters: the symptoms of depression and anxiety; level of social support; and the Dembo-Rubinstein technique in the general sample, all divided between men and women.

Table 1

Descriptive statistics

\begin{tabular}{lccc}
\hline & M/SD & M/SD Male & M/SD Female \\
\hline Depression & $7.48 / 6.02$ & $6.32 / 6.2$ & $8.77 / 5.59$ \\
Somatic sub-scale & $4.01 / 3.25$ & $3.07 / 3.09$ & $5.07 / 3.13$ \\
\hline Cognitive-affective sub-scale & $3.72 / 3.77$ & $3.46 / 3.87$ & $4.02 / 3.67$ \\
Anxiety & $4.75 / 5.41$ & $4.37 / 5.82$ & $5.18 / 4.92$ \\
\hline Social support & $39.14 / 6.91$ & $38.11 / 7.84$ & $40.03 / 5.54$ \\
Satisfaction with social support & $6.23 / 2.31$ & $5.85 / 2.04$ & $6.67 / 2.15$ \\
\hline Dembo-Rubinstein scales & & & \\
\hline DR Health & $7.22 / 1.73$ & $7.55 / 1.68$ & $6.85 / 1.73$ \\
\hline DR Luck & $6.93 / 1.91$ & $6.94 / 1.99$ & $6.93 / 1.83$ \\
DR Stress tolerance & $7.12 / 2.05$ & $7.46 / 1.95$ & $6.75 / 2.13$ \\
\hline DR Happiness & $7.65 / 2.14$ & $7.78 / 2.25$ & $7.52 / 2.02$ \\
\hline DR Sociability & $7.69 / 2.07$ & $7.85 / 2.44$ & $7.52 / 1.86$ \\
\hline
\end{tabular}

It is important to note that the average level of symptoms of anxiety based on cut-off scores exceeded the minimum values and fell into the group of "moderate" anxiety. Thus, $25.4 \%$ of respondents show moderate or higher levels of anxiety; $11.43 \%$ had a high level of anxiety; $24.13 \%$ had a mild level of depression or higher (also based on cut-off scores), and $6.35 \%$ of all participants had symptoms of moderate depression. 
Table 2 shows the main descriptive statistics of cognitive emotion regulation strategies.

Table 2

Descriptive statistics

\begin{tabular}{lcc}
\hline & M & SD \\
\hline CERQ Self-blame & 10.17 & 4.29 \\
CERQ Acceptance & 12.75 & 3.38 \\
\hline CERQ Rumination & 9.22 & 3.8 \\
\hline CERQ Positive refocusing & 13.38 & 3.91 \\
\hline CERQ Refocus on planning & 12.48 & 3.33 \\
CERQ Positive reappraisal & 13.18 & 3.95 \\
\hline CERQ Putting into perspective & 11.97 & 3.48 \\
\hline CERQ Catastrophizing & 7.7 & 2.96 \\
\hline CERQ Blaming others & 7.59 & 3.55 \\
\hline
\end{tabular}

As can be noted from Table 2, all available strategies are represented to one degree or another, showing that all of them were used by different patients to cope with the situation of the disease. We performed a factor analysis using the main components with orthogonal Varimax rotation. Factors were extracted based on eigenvalues greater than 1 . The resulting factor solution explained $69 \%$ of the dispersion. The results of the factor analysis are shown in Table 3.

Table 3

Factor analysis

\begin{tabular}{lccc}
\hline & \multicolumn{3}{c}{ Component } \\
\hline & $\mathbf{1}$ & $\mathbf{2}$ & $\mathbf{3}$ \\
\hline CERQ Refocus on planning & 0.836 & & \\
CERQ Positive reappraisal & 0.791 & & \\
CERQ Putting into perspective & 0.701 & & \\
CERQ Positive refocusing & 0.685 & & \\
CERQ Acceptance & 0.619 & & \\
CERQ Rumination & & 0.865 & -0.537 \\
CERQ Catastrophizing & & 0.745 & 0.905 \\
CERQ Self-blame & & & \\
CERQ Blaming others & & & \\
\hline
\end{tabular}


Internal consistency was assessed for each of the factors using the Cronbach's alpha calculation $(0.782,0.669$, and 0.801 , respectively). This way, we were able to single out three patterns of emotional coping strategies. The first one we named "adaptive cognitive change," which is characterized by the use of strategies such as refocusing on planning, positive reappraisal, putting into perspective, positive refocusing, and acceptance. We named the second pattern "fixation on negative experiences;" the most characteristic uses of this strategy are rumination and catastrophizing. The third category was "deflection of responsibility," and was characterized by the use of prosecution strategies - self-blame and blaming others.

Then we compared the different groups of participants according to the parameters studied in Table 1. Significant differences between the male and female groups were obtained using Student's t-distribution. The presence of statistically significant differences suggested that women have higher symptoms of depression than men $(\mathrm{t}=-2.31$, $\mathrm{p}$-value $<0.05)$, as well as the somatic component of depression $(\mathrm{t}=-3.4$, $\mathrm{p}$-value $<0.01)$. Moreover, women were more satisfied than men with the social support they received $(t=-2, p$-value $<0.05)$; also they assess their health lower than men, $(t=2.31$, $\mathrm{p}$-value $<0.01)$. No differences between men and women were found in the use of the emotion regulation patterns we identified, or in the use of individual strategies.

It is very important to emphasize that ANOVA showed no statistically significant differences between participants of different age groups. We initially assumed that both the emotion regulation strategies and the specifics of social support and anxiety (including about one's health) would differ in people of different ages.

An ANOVA test was performed for each of the three factors during the different time periods. Significant differences were obtained only for factor 1, adaptive cognitive change $(\mathrm{F}=4.793, \mathrm{p}=0.01)$. A Tamhane post-hoc test showed significant differences between stages 1 and 2, as well aso between stages 2 and $3(0.0027$ and 0.0031 respectively). Also ANOVA confirmed significant differences in strategies of cognitive emotional regulation between groups of respondents hospitalized at different stages of the pandemic. The use of such emotion regulation strategies as refocus on planning and positive reappraisal significantly increased when we moved to each new time period (e.g., refocus on planning went from $\mathrm{M}=10.66$, to $M=12.24$, to $M=13.54$; positive reappraisal went from $M=12$, to $M=12.55$, to $\mathrm{M}=14.66)$.

Table 4

Correlations between factors

\begin{tabular}{lcccc}
\hline & Depression & $\begin{array}{c}\text { Cognitive- } \\
\text { affective sub-scale }\end{array}$ & $\begin{array}{c}\text { Somatic } \\
\text { sub-scale }\end{array}$ & Anxiety \\
\hline Adaptive cognitive change & $-0.197^{*}$ & -0.111 & $-0.259^{*}$ & -0.111 \\
Fixation on negative experiences & $0.311^{* *}$ & $0.211^{*}$ & $0.345^{* *}$ & $0.350^{* *}$ \\
Deflection of responsibility & $0.178^{*}$ & 0.122 & $0.219^{*}$ & 0.012 \\
\hline
\end{tabular}

Note. ${ }^{*}$ means $p$-value $<0.01 ;{ }^{*}$ means $p$-value $<0.05$, 
We performed correlation analysis using the Spearman coefficient between the three patterns of emotional coping strategies and the symptoms of depression and anxiety. Regression coefficients were used as factor estimates. The results are shown in Table 4.

Thereafter, significant positive correlations were found between symptoms of depression and anxiety and coping with fixation on negative experiences (this is also true for the cognitive-affective component of depression). For other strategies, associations with symptoms of anxiety and depression were not significant.

Next, we performed a correlation analysis using Spearman's rank correlation coefficient to estimate the association between the social support, Dembo-Rubinstein sub-scales, emotion regulation strategies, and symptoms of depression and anxiety. The results are shown in Table 5.

Table 5

Correlations between scales

\begin{tabular}{lcccc}
\hline & Depression & $\begin{array}{c}\text { Somatic } \\
\text { sub-scale }\end{array}$ & $\begin{array}{c}\text { Cognitive-affective } \\
\text { sub-scale }\end{array}$ & Anxiety \\
\hline Social support & $-0.302^{* *}$ & $-0.289^{* *}$ & $-0.263^{* *}$ & -0.136 \\
Satisfaction with social support & -0.138 & -0.061 & $-0.194^{*}$ & -0.134 \\
CERQ Self-blame & 0.007 & 0.003 & -0.009 & 0.072 \\
CERQ Acceptance & -0.017 & 0.0008 & -0.015 & 0.07 \\
CERQ Rumination & $0.348^{* *}$ & $0.271^{* *}$ & $0.342^{* *}$ & $0.458^{* *}$ \\
CERQ Positive refocusing & $-0.278^{* *}$ & $-0.180^{*}$ & $-0.330^{* *}$ & $-0.221^{*}$ \\
CERQ Refocus on planning & -0.136 & -0.078 & $-0.181^{*}$ & 0.072 \\
CERQ Positive reappraisal & $-0.280^{*}$ & $-0.208^{*}$ & $-0.313^{* *}$ & -0.133 \\
CERQ Putting into perspective, & 0.026 & 0.044 & -0.018 & -0.039 \\
CERQ Catastrophizing & $0.226^{*}$ & 0.122 & $0.270^{* *}$ & $0.186^{*}$ \\
CERQ Blaming others & $0.207^{*}$ & 0.136 & $0.257^{* *}$ & 0.077 \\
DR Health & $-0.396^{* *}$ & $-0.454^{* *}$ & $-0.265^{* *}$ & -0.103 \\
DR Stress tolerance & $-0.440^{* *}$ & $-0.441^{* *}$ & $-0.368^{* *}$ & $-0.346^{* *}$ \\
DR Happiness & $-0.416^{* *}$ & $-0.409^{* *}$ & $-0.321^{* *}$ & $-0.251^{* *}$ \\
DR Sociability & $-0.307^{* *}$ & $-0.317^{* *}$ & $-0.260^{* *}$ & $-0.183^{*}$ \\
DR Luck & $-0.331^{* *}$ & $-0.276^{* *}$ & $-0.356^{* *}$ & -0.036 \\
\hline
\end{tabular}

Note. ${ }^{* *}$ means $p$-value $<0.01, n=127 ;{ }^{*}$ means $p$-value $<0.05, n=127$

Note that the severity of symptoms of depression was positively associated with the use of an emotion regulation strategy such as rumination, and negatively associated with the use of strategies such as positive refocusing and positive reap- 
praisal. Also, the severity of symptoms of depression was negatively associated with perceived social support, as well as with all the scales in the Dembo-Rubinstein technique: the more points on the depression scale, the less healthy, lucky, stressresistant, happy, and sociable respondents would see themselves. It is also important to note that these patterns were valid for both somatic and cognitive-affective components. The severity of the symptoms of anxiety was also positively associated with the use of a rumination strategy to cope with COVID-19, and negatively associated with the assessment of oneself as less stressful.

\section{Discussion}

Our data suggest that symptoms of depression and anxiety are indeed characteristic of hospitalized COVID-19 patients. These traits are significantly associated with patients' perceptions of being less healthy, fortunate, and happy, and more stressed. The prevalence of these traits in our series of results turned out to be less than in other papers (Salari et al., 2020), which may be due to less severe COVID-19 symptoms in our patients, or to delayed symptoms of depression and anxiety (since the patients assessed their condition only 10-14 days after hospitalization and diagnosis, they may have shown more symptoms later on in their disease).

Our results showed that the severity of depression and anxiety symptoms among hospitalized COVID-19 patients was positively associated with the use of emotion regulation strategies aimed at fixation on negative experiences, rumination, and catastrophizing. These results are supported by a number of other studies (Garnefski et al., 2002; Koole et al., 1999). At the same time, the use of other strategies, such as deflection of responsibility and adaptive cognitive change, did not reduce the severity of depression and anxiety at a statistically significant level.

According to other studies, strategies such as putting into perspective, positive reappraisal, and refocusing on planning are associated with a significant reduction in the severity of depression, anxiety, and stress (Martin \& Dahlen, 2005; Garnefski et al., 2002). As we have already noted above, it is not legitimate to say that strategies are effective without taking into account the context in which they are being implemented. And in a situation of emergency hospitalization with COVID-19, according to our data, these strategies are rather unproductive in coping with depression and anxiety.

Our initial hypothesis stating that the regulatory strategies used would differ among research participants of different ages was not confirmed. Coupled with our finding that there are no significant differences in the use of certain regulatory strategies between men and women, we can say that the patterns of emotional coping strategies are primarily due to the emergence of a new, unknown disease (COVID-19) rather than being related to the previous experience of the respondents.

The lack of differences in intensity of the majority of the strategies used by the hospitalized patients at various stages of the pandemic, and the negative correlations between these strategies and the severity of depression allow us to assume that there are still no unambiguous and clear ways to cope successfully with news of a COVID-19 diagnosis. Now the task of finding such ways of coping and imple- 
menting psychotherapeutic programs that help patients deal with hospitalization and the fact of their illness falls to clinical psychologists and psychiatrists. When preparing such programs, one can also take into account the fact that a number of the strategies included in the "adaptive cognitive change" pattern nevertheless become more common in the later stages of the the studied period. Perhaps, after the general uncertainty in society caused by the COVID-19 pandemic diminishes, these strategies will become promising in coping with the disease.

Our results on the differences between the male and female participants coincide with the data of foreign colleagues and with general trends: women expressed more symptoms of depression (Cavanagh, et al., 2017). The fact that women rated their health as more severe turned out to be quite interesting. A number of authors (Racine et al., 2012) indicate that women have lower sensitivity to pain, somatic manifestations, and symptoms of bodily discomfort. Other studies show that women tend to report more physical symptoms than men (Barsky et al., 2001; Van Wijk, C.M.T.G., \& Kolk, A.M., 1997). Unfortunately, due to certain limitations, we did not conduct interviews with patients after using the Dembo-Rubinstein technique. Therefore, we do not know what criteria the patients relied on when assessing their well-being according to the "Health" sub-scale. However, we found no data describing differences in the subjective experience of COVID-19 symptoms between women and men.

Our results on social support coincide with data from other studies. Such support is more associated with a reduction in depressive symptoms than instrumental support is (Gariépy et al., 2016; Yoo et al., 2017). But, when talking about the situation with COVID-19, it is important to understand that social support cannot, and should not, become the key protective factor against depressive symptoms for patients. This is primarily due to the fact that for patients under quarantine, and hospitalized in isolation chambers, access to potential support from relatives and patient-care specialists is necessarily sharply limited: mobile phone and Internet access become the only ways to receive such support.

\section{Conclusion}

It is obvious that the very existence of the COVID-19 pandemic has greatly transformed the routine of a resident of the metropolis and imposed a number of complex and unusual restrictions. For those who at this moment are not only witnesses to the pandemic, but also sick, the situation seems even more difficult. Our study showed that patients with COVID-19 have symptoms of depression and anxiety, which is consistent with data from patients from other countries; women show symptoms of depression more than men.

The patterns of emotion regulation strategies most commonly used by our respondents to cope with the disease, turned out to be insufficiently effective in relieving symptoms of depression and anxiety; the pattern called "adaptive cognitive change" was only marginally associated with a decrease in symptoms of depression and anxiety. To the contrary, one of the identified factors - fixation on negative experiences - significantly impaired a patient's psychological well-being. This means that, since the beginning of the pandemic and despite the rather long duration of the research, the patients have not had highly effective ways available to cope with 
what is happening to them. This challenges specialists, doctors, and psychologists with the task of developing psychological support programs for COVID-19 patients and their loved ones. We assume that such programs in general can be aimed at reducing the use of strategies included in the patterns "deflection of responsibility" and "fixation on negative experiences," and strengthening and practicing the strategies included in the pattern "adaptive cognitive change."

In further studies, it seems important to continue to highlight buffer factors that prevent the formation of depressive and anxious reactions to the disease, as well as to study the formation of the most productive strategies for regulating emotional states in this particular situation.

\section{Limitations}

Our research was conducted at a Moscow clinic, and all respondents who took part in the work lived in Moscow. This makes it difficult to apply the data obtained to a larger sample and does not allow us to conclude how residents of other cities might cope emotionally with the COVID-19 situation. Due to the remote format of the study (by internet), we have no data on the severity of the patients' somatic symptoms. This prevents us from investigating the important relationship between a patient's physical condition and the severity of his or her symptoms of depression and anxiety.

Also, the number of participants in the three groups of patients hospitalized at different stages of the pandemic turned out to be unequal due to organizational limitations in the work of clinics. This also does not allow us to unambiguously generalize the results obtained.

According to our assumptions, another possible important limitation is that the patients who agreed to undergo the online research felt significantly better physically than those who, for health reasons, could not take part in the survey. This may mean that the patterns of coping with the disease identified by us are fair only for those patients who, on the one hand, have enough physiological symptoms for hospitalization, but, on the other hand, feel good enough for voluntary participation in psychological work. Therefore, we cannot unequivocally assert how asymptomatic patients or patients with severe symptoms of COVID-19 are dealing with the disease.

\section{References}

Barsky, A.J., Peekna, H.M., \& Borus, J.F. (2001). Somatic symptom reporting in women and men. Journal of General Internal Medicine, 16(4), 266-275. https://doi.org/10.1046/j.15251497.2001.016004266.x

Beck, A.T., Steer, R.A., \& Brown, G.K. (1996). Manual for the Beck Depression Inventory-II. San Antonio, TX: Psychological Corporation. https://doi.org/10.1037/t00742-000

Cavanagh, A., Wilson, C.J., Kavanagh, D.J., \& Caputi, P. (2017). Differences in the Expression of Symptoms in Men Versus Women with Depression. Harvard Review of Psychiatry, 25(1), 29-38. https://doi.org/10.1097/HRP.0000000000000128

Domínguez-Salas, S., Gómez-Salgado, J., Andrés-Villas, M., Díaz-Milanés, D., Romero-Martín, M., \& Ruiz-Frutos, C. (2020). Psycho-Emotional Approach to the Psychological Distress Related to the COVID-19 Pandemic in Spain: A Cross-Sectional Observational Study. Healthcare, 8(3), 190. https://doi.org/10.3390/healthcare8030190 
Epstein, D., Andrawis, W., Lipsky, A., Ziad, H., \& Matan, M. (2020). Anxiety and Suicidality in a Hospitalized Patient with COVID-19 Infection. European Journal of Case Reports in Internal Medicine, 7(5). https://doi.org/10.12890/2020_001651

Gariépy, G., Honkaniemi, H., \& Quesnel-Vallée, A. (2016). Social support and protection from depression: systematic review of current findings in Western countries. British Journal of Psychiatry, 209(04), 284-293. https://doi.org/10.1192/bjp.bp.115.169094

Garnefski, N., \& Kraaij,V. (2007). The Cognitive Emotion Regulation Questionnaire: Psychometric features and prospective relationships with depression and anxiety in adults. European Journal of Psychological Assessment, 23, 141-149. https://doi.org/10.1027/1015-5759.23.3.141

Garnefski, N., Kraaij, V., \& Spinhoven, P. (2001). Negative life events, cognitive emotion regulation and emotional problems. Personality and Individual Differences, 30(8), 1311-1327. https://doi. org/10.1016/S0191-8869(00)00113-6

Garnefski, N., Van Den Kommer, T., Kraaij, V., Teerds, J., Legerstee, J., \& Onstein, E. (2002). The relationship between cognitive emotion regulation strategies and emotional problems: comparison between a clinical and a non-clinical sample. European Journal of Personality, 16(5), 403-420. https://doi.org/10.1002/per.458

Gross, J.J. (2013). Emotion regulation: Taking stock and moving forward. Emotion, 13(3), 359-365. https://doi.org/10.1037/a0032135

Gross, J.J., \& Thompson, R.A. (2007). Emotion Regulation: Conceptual Foundations. In J.J. Gross (Ed.), Handbook of Emotion Regulation (pp. 3-24). The Guilford Press.

Holmogorova, A.B., Garanyan, N.G., \& Petrova, G.A. (2003). Social support as a subject of scientific study and its impairment in patients with affective spectrum disorders. Soc. i klin. psihiatriya, 2, $15-23$.

Koole, S.L., Smeets, M., van Knippenberg, A., \& Dijksterhuis, A. (1999). The cessation of rumination through self-affirmation. Journal of Personality and Social Psychology, 77, 111-125. https://doi. org/10.1037/0022-3514.77.1.111

Martin, R.C., \& Dahlen, E.R. (2005). Cognitive emotion regulation in the prediction of depression, anxiety, stress, and anger. Personality and Individual Differences, 39(7), 1249-1260. https://doi. org/10.1016/j.paid.2005.06.004

Petzold, M.B., Bendau, A., Plag, J., Pyrkosch, L., Mascarell Maricic, L., Betzler, F., .. Ströhle, A. (2020). Risk, resilience, psychological distress, and anxiety at the beginning of the COVID-19 pandemic in Germany. Brain and Behavior. 9(10), e01745. https://doi.org/10.1002/brb3.1745

Racine, M., Tousignant-Laflamme, Y., Kloda, L.A., Dion, D., Dupuis, G., \& Choinière, M. (2012). A systematic literature review of 10 years of research on sex/gender and pain perception - Part 2: Do biopsychosocial factors alter pain sensitivity differently in women and men? Pain, 153(3), 619-635. https://doi.org/10.1016/j.pain.2011.11.026

Rajkumar, R.P. (2020). COVID-19 and mental health: A review of the existing literature. Asian Journal of Psychiatry, 102066. https://doi.org/10.1016/j.ajp.2020.102066

Rasskazova, E.I., Leonova, A.B., \& Pluzhnikov, I.V. (2011). Razrabotka russkoiazychnoi versii oprosnika kognitivnoi reguliatsii emotsii [Development of the Russian-language version of the cognitive emotional regulation questionnaire]. Vestnik Moskovskogo Universiteta. Seriia 14. Psikhologiia [Moscow University Psychology Bulletin], 4, 161-179.

Renjun, G., Ziyun, L., Xiwu, Y., Wei, W., Yihuang, G., Chunbing, Z., \& Zhiguang, S. (2020). Psychological intervention on COVID-19: a protocol for systematic review and meta-analysis. Medicine, 21(99), e20335. https://doi.org/10.1097/MD.0000000000020335

Rubinshtejn, S.Ya. (2010). Eksperimentalnye metodiki patopsikhologii i opyt primeneniya ikh $v$ klinike [Experimental methods of pathopsychology and experience of their application in the clinic]. Moscow, Aprel'-Press.

Salari, N., Hosseinian-Far, A., Jalali, R., Vaisi-Raygani, A., Rasoulpoor, S., Mohammadi, M., \& Khaledi-Paveh, B. (2020). Prevalence of stress, anxiety, depression among the general population during the COVID-19 pandemic: a systematic review and meta-analysis. Globalization and Health, 16(1). https://doi.org/10.1186/s12992-020-00589-w 
Sapolsky, R.M. (2007). Stress, Stress-Related Disease, and Emotional Regulation. In J.J. Gross (Ed.), Handbook of Emotion Regulation (pp. 606-615). The Guilford Press.

Spitzer, R.L., Kroenke, K., Williams, J.B., \& Löwe, B. (2006). A brief measure for assessing generalized anxiety disorder: the GAD-7. Arch Intern Med., 166(10), 1092-1097. https://doi.org/10.1001/ archinte.166.10.1092

Sher, L. (2020). The impact of the COVID-19 pandemic on suicide rates. QJM: An International Journal of Medicine, 10(113), 707-712. https://doi.org/10.1093/qjmed/hcaa202

Tarabrina, N.V. (2001) Praktikum po psihologii posttravmaticheskogo stressa. [Workshop on the psychology of posttraumatic stress]. St. Petersburg: Peter.

Thompson, R.A. (2008). A Theme in Search of Definition. Monographs of the Society for Research in Child Development, 59(2-3), 25-52. https://doi.org/10.1111/j.1540-5834.1994.tb01276.x

Van Wijk, C.M.T.G., \& Kolk, A.M. (1997). Sex differences in physical symptoms: The contribution of symptom perception theory. Social Science \& Medicine, 45(2), 231-246. https://doi.org/10.1016/ S0277-9536(96)00340-1

Wang, C., Pan, R., Wan, X., Tan, Y., Xu, L., McIntyre, R.S., \& Ho, C. (2020). A longitudinal study on the mental health of general population during the COVID-19 epidemic in China. Brain, Behavior, and Immunity, 87, 40-48. https://doi.org/10.1016/j.bbi.2020.04.028

Wei, N., Huang, B., Lu, S., Hu, J., Zhou, X., Hu, C., ... Hu, S. (2020). Efficacy of internet-based integrated intervention on depression and anxiety symptoms in patients with COVID-19. Journal of Zhejiang University-SCIENCE B. https://doi.org/10.1631/jzus.B2010013

Xiang, Y.-T., Yang, Y., Li, W., Zhang, L., Zhang, Q., Cheung, T., \& Ng, C.H. (2020). Timely mental health care for the 2019 novel coronavirus outbreak is urgently needed. The Lancet Psychiatry. https://doi.org/10.1016/S2215-0366(20)30046-8

Yoo, H., Shin, D.W., Jeong, A., Kim, S.Y., Yang, H., Kim, J.S., .. Park, J.-H. (2017). Perceived social support and its impact on depression and health-related quality of life: a comparison between cancer patients and the general population. Japanese Journal of Clinical Oncology, 47(8), 728-734. https://doi.org/10.1093/jjco/hyx064

Yuan, B., Li, W., Liu, H., Cai, X., Song, S., Zhao, J., ... An, Y. (2020). Correlation between immune response and self-reported depression during convalescence from COVID-19, Brain, Behaviour, and Immunity, 88, 39-43. https://doi.org/10.1016/j.bbi.2020.05.062

Original manuscript received July 12, 2020

Revised manuscript accepted November 15, 2020

First published online December 30, 2020

To cite this article: Dovbysh, D.V., Kiseleva, M.G. (2020). Cognitive Emotion Regulation, Anxiety, and Depression in Patients Hospitalized with COVID-19. Psychology in Russia: State of the Art, 13(4), 134-147. DOI: 10.11621/pir.2020.0409 